\title{
Força de preensão manual e aptidões físicas: um estudo preditivo com idosos ativos
}

\author{
Grip strength and physical fitness: a predictive study with active elderly
}

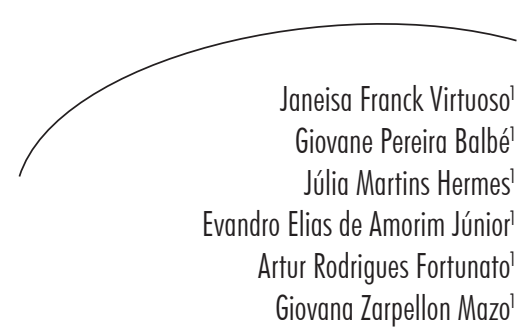

Resumo

Introdução: A avaliação da força de preensão manual (FPM) é um método útil para avaliar a força muscular global de pessoas idosas. Objetivo: Verificar a relação entre a FPM e as aptidões físicas de idosos praticantes de exercícios físicos, segundo o sexo. Métodos: Estudo descritivo com 106 idosas e 30 idosos praticantes de exercícios físicos num programa de extensão universitário, que foram submetidos a bateria Senior Fitness Test (força dos membros inferiores, força dos membros superiores, flexibilidade dos membros inferiores, agilidade/equilíbrio dinâmico, flexibilidade dos membros superiores e resistência aeróbica). A FPM foi avaliada por um dinamômetro de preensão manual. Utilizou-se estatística descritiva (média e desvio-padrão) e inferencial por meio do teste U de Mann Whitney para amostras independentes e regressão linear simples. O nível de significância foi 5\%. Resultados: A FPM direita e esquerda foi maior nos homens do que nas mulheres $(\mathrm{p}<0,001)$. Observou-se que a FPM é boa preditora da força de membros superiores (aproximadamente 11,4\%) e a resistência aeróbica (aproximadamente 14,0\%) nas mulheres, enquanto nos homens a FPM explica essas aptidões (aproximadamente $22,0 \%$ e $20,0 \%$, respectivamente) e também a agilidade/equilíbrio (aproximadamente, 21,0\%). Conclusões: Observa-se que, entre idosos praticantes de exercícios físicos, a FPM prediz melhor apenas a variação de testes físicos que apresentam bom desempenho, o que aumenta o poder de explicação e predição entre os homens, já que estes obtiveram melhores desempenhos nas avaliações físicas.

\section{Abstract}

Introduction: Evaluation of handgrip strength (HS) is a useful method for assessing overall muscle strength of old people. Objective: To investigate the relationship between HS and the physical skills of old people practitioners of physical exercise, according to sex. Methods: A descriptive study with 106 women and 30 man practicing physical exercises in a university extension program, which underwent a battery of Senior Fitness Test (lower limb strength, upper limbs strength, lower limbs flexibility, agility

Palavras-chave: Força da Mão. Aptidão Física. Idoso.

\footnotetext{
Curso de Educação Física, Centro de Ciências da Saúde e do Esporte. Universidade do Estado de Santa Catarina. Florianópolis, SC, Brasil.
} 
/ dynamic balance, upper limb flexibility and aerobic endurance). HS was evaluated by a handgrip dynamometer. We used descriptive statistics (mean and standard deviation) and inferential $U$ test of Mann Whitney tests for independent samples and simple linear regression. The significance level was 5\%. Results: The HS right and left was higher in men than in women $(\mathrm{p}<0.001)$. It was observed that HS is a good predictor of upper limb strength (approximately 11.4\%) and aerobic endurance (approximately 14.0\%) in women, while in men, HS explains these skills (approximately $22.0 \%$ and $20.0 \%$, respectively) and also the agility / dynamic balance (approximately $21.0 \%$ ). Conclusions: We observed that, among elderly practicing physical exercises, HS better predicts only the variation of physical tests that perform well, which increases the power of explanation and prediction among men, as they had better performance in physical assessments.
Key words: Hand Strength. Physical Fitness. Elderly.

\section{INTRODUÇÃO}

A força de preensão manual (FPM) é um dos elementos básicos na análise das capacidades manipulativas, de força e de movimentos da mão. Para Sasaki et al., ${ }^{1}$ a FPM é muito útil para avaliar a força muscular global de pessoas de meia-idade e idosos, sendo também um instrumento simples e bom preditor de prognóstico de saúde. Além disso, a FPM permite analisar o desempenho funcional, à medida que as pessoas envelhecem, ${ }^{2}$ pois a perda no sistema muscular está relacionada à mudança no desempenho neuromuscular, que pode ser verificada pela lentificação dos movimentos, perda da força muscular e fadiga muscular precoce. ${ }^{3}$ Essas mudanças podem influenciar no equilíbrio, flexibilidade e velocidade das ações musculares, sugerindo que, além da força muscular, a FPM também permite predizer outras aptidões. ${ }^{4}$

Nesse sentido, uma pesquisa ${ }^{5}$ aponta que a FPM é boa preditora do desempenho em tarefas motoras de idosos residentes em instituições asilares. Entretanto, nota-se carência de estudos que investiguem se a FPM é um indicador global da força em idosos praticantes de exercícios físicos. Outro aspecto que não tem recebido devida atenção no estudo da FPM em idosos é a possível influência do sexo nessa variável, uma vez que a FPM dos homens é superior, justificada pela maior massa e força muscular global quando comparado às mulheres. ${ }^{6-8}$
Desse modo, o objetivo do presente estudo foi verificar a relação entre a força de preensão manual e as aptidões físicas relacionadas à saúde de idosos praticantes de exercícios físicos, segundo o sexo.

\section{MÉTODOS}

\section{Tipo de estudo e casuística}

Para este estudo, selecionaram-se idosos participantes dos projetos de exercícios físicos do Programa de Extensão Grupo de Estudos da Terceira Idade (GETI) da Universidade do Estado de Santa Catarina (UDESC), cuja população é de 300 idosos, aproximadamente. A maioria dos idosos pratica mais de uma modalidade, sendo que $44,5 \%$ realizavam hidroginástica; 30,1\%, natação; e 39,3\%, ginástica. As aulas de exercício físico dos programas têm duração de 50 minutos e são realizadas de duas a três vezes por semana, com intensidade moderada.

A amostra foi selecionada de forma intencional, tendo como critérios de inclusão: serem idosos ( $\geq 60$ anos de idade) e praticantes de exercícios físicos no GETI há, no mínimo, seis meses. Para determinar o tamanho amostral, utilizou-se um tamanho de efeito $\left(\mathrm{f}^{2}\right)$ médio igual a 0,15 , probabilidade de erro de 0,05 e força de $80 \%$. Dessa forma, o número mínimo de idosos, neste estudo com sete preditores, foi de 103. Diante desses critérios, fizeram parte do estudo 136 idosos, sendo 106 mulheres e 30 homens. 
Instrumentos

Para identificar o sexo, idade e tempo de prática de exercício físico no GETI, foi aplicada uma entrevista aos idosos. E com o objetivo de investigar a aptidão física dos idosos integrantes do estudo, utilizou-se a bateria de testes de aptidão física para idosos Senior Fitness Test (SFT), ${ }^{9}$ pois é de fácil aplicação e baixo custo operacional, ${ }^{10}$ além de ser validada para a população idosa. ${ }^{11}$
Essa bateria é composta por seis testes físicos: levantar da cadeira (força dos membros inferiores), flexão de braço (força dos membros superiores), sentar e alcançar os pés (flexibilidade dos membros inferiores), levantar e caminhar (agilidade e o equilíbrio dinâmico), alcançar as costas mão direita e esquerda (flexibilidade dos membros superiores) e caminhada de seis minutos (resistência aeróbica), segundo o quadro 1.

Quadro 1. Protocolo de utilização dos testes de aptidão física da bateria Senior Fitness Test, aplicados a amostra de idosos praticantes de exercícios físicos, adaptado de Rikli \& Jones. ${ }^{9}$ Florianópolis-SC, 2011.

\begin{tabular}{|c|c|c|c|}
\hline Teste & Objetivo & Descrição & Pontuação \\
\hline $\begin{array}{l}\text { Levantar da } \\
\text { cadeira }\end{array}$ & $\begin{array}{l}\text { Avaliar a força } \\
\text { dos membros } \\
\text { inferiores }\end{array}$ & $\begin{array}{l}\text { Ao sinal positivo, o participante, que está na } \\
\text { posição sentada, ergue-se e fica totalmente em pé } \\
\text { e então retorna à posição sentada. }\end{array}$ & $\begin{array}{l}\text { Registra-se o número total } \\
\text { de repetições num intervalo } \\
\text { de } 30 \text { segundos. }\end{array}$ \\
\hline $\begin{array}{l}\text { Flexão de } \\
\text { braço }\end{array}$ & $\begin{array}{l}\text { Avaliar a força } \\
\text { dos membros } \\
\text { superiores }\end{array}$ & $\begin{array}{l}\text { O participante estará na posição sentada, } \\
\text { braço dominante estendido e perpendicular ao } \\
\text { chão, segurando um halter. Ao sinal positivo, } \\
\text { o participante gira sua palma para cima } \\
\text { enquanto flexiona o braço em amplitude total } \\
\text { de movimento e então retorna o braço para uma } \\
\text { posição estendida. Utilizou-se um halter de } 2 \mathrm{~kg} \\
\text { para mulheres e } 4 \mathrm{Kg} \text { para homens. }\end{array}$ & $\begin{array}{l}\text { Registra-se o número total } \\
\text { de repetições num intervalo } \\
\text { de } 30 \text { segundos. }\end{array}$ \\
\hline $\begin{array}{l}\text { Sentar e } \\
\text { alcançar os pés }\end{array}$ & $\begin{array}{l}\text { Avaliar a } \\
\text { flexibilidade } \\
\text { dos membros } \\
\text { inferiores }\end{array}$ & $\begin{array}{l}\text { O participante sentado e com uma perna } \\
\text { estendida, inclina-se lentamente para frente. } \\
\text { O avaliado tenta tocar os dedos dos pés } \\
\text { escorregando as mãos, uma em cima da outra, } \\
\text { com as pontas dos dedos médios, na perna } \\
\text { estendida. }\end{array}$ & $\begin{array}{l}\text { Registra-se a distância } \\
(\mathrm{cm}) \text { até os dedos dos pés } \\
\text { (resultado mínimo) ou } \\
\text { a distância (cm) que se } \\
\text { consegue alcançar para } \\
\text { além dos dedos dos pés } \\
\text { (resultado máximo). }\end{array}$ \\
\hline $\begin{array}{l}\text { Levantar e } \\
\text { caminhar }\end{array}$ & $\begin{array}{l}\text { Avaliar agilidade } \\
\text { e o equilíbrio } \\
\text { dinâmico }\end{array}$ & $\begin{array}{l}\text { Ao sinal indicativo, o avaliado levanta da cadeira, } \\
\text { caminha o mais rapidamente possível em volta } \\
\text { de um cone, retorna para a cadeira e senta. O } \\
\text { cone distancia-se da cadeira em } 2,44 \text { metros. }\end{array}$ & $\begin{array}{l}\text { Registra-se o tempo } \\
\text { decorrido entre o sinal de } \\
\text { "partida" até o momento em } \\
\text { que o participante senta-se } \\
\text { novamente na cadeira. }\end{array}$ \\
\hline $\begin{array}{l}\text { Alcançar as } \\
\text { costas mão } \\
\text { direita e } \\
\text { esquerda }\end{array}$ & $\begin{array}{l}\text { Avaliar a } \\
\text { flexibilidade } \\
\text { dos membros } \\
\text { superiores }\end{array}$ & $\begin{array}{l}\text { Em pé, o participante coloca a mão sobre } \\
\text { o mesmo ombro, a palma aberta e os dedos } \\
\text { estendidos, alcançando o meio das costas. A mão } \\
\text { do outro braço está colocada atrás das costas, a } \\
\text { palma aberta, alcançando para cima na tentativa } \\
\text { de tocar ou sobrepor os dedos médios estendidos. }\end{array}$ & $\begin{array}{l}\text { Registra-se a distância entre } \\
\text { as pontas dos dedos (-) ou } \\
\text { a distância da sobreposição } \\
\text { (+), em centímetros. }\end{array}$ \\
\hline $\begin{array}{l}\text { Caminhada de } \\
\text { seis minutos }\end{array}$ & $\begin{array}{l}\text { Avaliar a } \\
\text { resistência } \\
\text { aeróbica. }\end{array}$ & $\begin{array}{l}\text { Ao sinal indicativo, o participante caminha } \\
\text { o mais rápido possível em volta do percurso, } \\
\text { quantas vezes puder, durante o tempo de seis } \\
\text { minutos. O teste utiliza um percurso de } 50 \mathrm{~m} \\
\text { medido dentro de segmentos de } 5 \mathrm{~m} \text {. }\end{array}$ & $\begin{array}{l}\text { Registra-se a distância, } \\
\text { (em metros) percorrida no } \\
\text { intervalo de seis minutos. }\end{array}$ \\
\hline
\end{tabular}


Para avaliação da força de preensão manual direita $\left(\mathrm{FPM}_{\mathrm{dir}}\right)$ e esquerda $\left(\mathrm{FPM}_{\mathrm{csq}}\right)$, empregouse o teste de força máxima de preensão manual, realizado por meio de um dinamômetro (Hydraulic Hand Dynamometer, modelo Sh5001), da Saehan Corporation. O teste de FPM foi realizado segundo as recomendações da Sociedade Americana de Terapeutas de Mão (SATM), em que o participante deve estar confortavelmente sentado, posicionado com o ombro aduzido, o cotovelo fletido a $90^{\circ}$, o antebraço em posição neutra, podendo a posição do punho variar de 0 a $30^{\circ}$ de extensão.

Foi solicitado que o indivíduo alcançasse a maior força possível em cada uma das mãos, sendo sempre realizadas, previamente, demonstração e familiarização com instrumento e o sistema do teste. ${ }^{12}$ O pico máximo foi registrado em quilograma-força ( $\mathrm{Kgf}$ ), tanto para mão direita, como para a esquerda, inferido a partir do cálculo da média aritmética de três medidas realizadas de cada lado, respeitando-se um período de 20 segundos de repouso entre duas medidas do mesmo lado. ${ }^{13}$

\section{Coleta de dados}

Os dados foram coletados em novembro de 2011 por alunos do CEFID/UDESC previamente treinados. Inicialmente, realizou-se contato pessoal com os idosos, explicando-se o objetivo da pesquisa, o sigilo da identificação e solicitando sua participação. Em seguida, foram agendados data, horário e local para aplicação dos testes de aptidão física e da avaliação da força de preensão manual. Antes da aplicação das avaliações, os idosos que concordaram em participar da pesquisa assinaram o Termo de Consentimento Livre e Esclarecido em duas vias, ficando uma via de posse do idoso e a outra do pesquisador.

Primeiramente, os idosos realizaram avaliação da força de preensão manual direita e esquerda e, em seguida, a bateria de testes de aptidão física para idosos Senior Fitness Test - SFT, conforme a ordem exposta no quadro 1.
Tratamento de dados

Os dados foram organizados no programa Excel ${ }^{\circledR}$ e analisados no software estatístico Statistical Package for Social Sciences (SPSS), versão 20.0 para Windows. A descrição dos dados ocorreu mediante apresentação de média e desvio-padrão. A comparação das variáveis "idade", "força de preensão manual direita e esquerda" e as aptidões físicas, segundo o sexo, se deu por meio do teste U de Mann Whitney. Foi realizada análise de regressão linear simples, para relacionar as aptidões físicas (variáveis dependentes) com a força de preensão manual direita e esquerda (variáveis independentes), estratificadas segundo sexo. Adotou-se um nível de significância de 5\%.

\section{Aspectos éticos}

A pesquisa foi conduzida dentro dos padrões exigidos pela Resolução nº. 196/96, do Conselho Nacional de Saúde, e aprovada pelo Comitê de Ética em Pesquisas em seres humanos da Universidade do Estado de Santa Catarina, sob o protocolo no $185 / 2007$.

\section{RESULTADOS}

A amostra deste estudo foi composta de 136 idosos praticantes de exercícios físicos com média

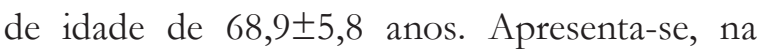
tabela 1, a média desvio-padrão e a comparação das variáveis "idade", "força de preensão manual direita" $\left(\mathrm{FPM}_{\mathrm{dir}}\right)$ e "esquerda" $\left(\mathrm{FPM}_{\text {esq }}\right)$ e os testes de aptidão física entre os sexos.

Observa-se ainda, na tabela 1, que a média da FPM, tanto da mão direita quanto da esquerda, é significantemente superior entre os homens (aproximadamente 30\%). Quanto aos testes físicos, aqueles que representam aptidões de flexibilidade como o de membros inferiores e superiores mostraram valores médios inferiores entre os homens. No entanto, a capacidade aeróbica mostrou-se inferior entre as mulheres. 
Tabela 1. Comparação das variáveis idade, força de preensão manual direita $\left(\mathrm{FPM}_{\mathrm{dir}}\right)$ e esquerda $\left(\mathrm{FPM}_{\mathrm{esq}}\right)$ e testes de aptidão física entre os sexos. Florianópolis-SC, 2011.

\begin{tabular}{lcccc}
\hline \multicolumn{1}{c}{ Variáveis } & $\begin{array}{c}\text { Homens } \\
(\mathrm{n}=30) \\
\text { média } \pm \text { dp }\end{array}$ & $\begin{array}{c}\text { Mulheres } \\
(\mathrm{n}=106) \\
\text { média } \pm \text { dp }\end{array}$ & $\mathrm{U}$ & $\mathrm{p}$ \\
\hline Idade (anos) & $70,4 \pm 6,7$ & $68,5 \pm 5,5$ & 1,55 & 0,122 \\
$\mathrm{FPM}_{\text {dir }}(\mathrm{kgf})$ & $35,2 \pm 9,0$ & $22,9 \pm 5,0$ & 7,15 & $<0,001^{* *}$ \\
$\mathrm{FPM}_{\text {esq }}$ (kgf) & $32,1 \pm 8,6$ & $21,0 \pm 4,3$ & 6,85 & $<0,001^{* *}$ \\
Força de membros inferiores (rep) & $13,7 \pm 3,1$ & $13 \pm 2,8$ & 1,20 & 0,232 \\
Força de membros superiores (rep) & $15,1 \pm 3,9$ & $14,6 \pm 4,1$ & 0,56 & 0,575 \\
Flexibilidade de membros inferiores (cm) & $-1,9 \pm 13,8$ & $4,4 \pm 9,5$ & $-2,32$ & $0,026^{*}$ \\
Agilidade/equilíbrio dinâmico (seg) & $5,6 \pm 1,0$ & $5,8 \pm 1,0$ & $-0,85$ & 0,396 \\
Flexibilidade do membro superior & $-9,6 \pm 8,1$ & $-5,6 \pm 8,7$ & $-2,24$ & $0,026^{*}$ \\
direito (cm) & $-13,7 \pm 9,0$ & $-8,42 \pm 8,8$ & $-2,89$ & $0,004^{*}$ \\
Flexibilidade do membro superior esquerdo $(\mathrm{cm})$ & $566,5 \pm 67,8$ & $515,8 \pm 71,1$ & 3,48 & $0,001^{*}$ \\
\hline Resistência aeróbica (m) & &
\end{tabular}

$\mathrm{FPM}_{\mathrm{dir}}=$ força de preensão manual direita; $\mathrm{FPM}_{\text {esq }}=$ força de preensão manual esquerda; $\mathrm{Kgf}=$ kilograma força; rep= repetição; seg= segundos; $\mathrm{cm}=$ centímetros; $\mathrm{m}=$ metros; $U=$ teste $U$ de Mann-Whitney; $\mathrm{p}=$ nível de significância; $\mathrm{dp}=$ desvio-padrão; ${ }^{\text {esq }}<0,05 ;{ }^{* *} \mathrm{p}<0,001$.

Nas tabelas 2 e 3, é possível observar a relação entre a FPM (direita e esquerda) e as aptidões físicas, as quais foram analisadas por meio da regressão linear simples, segundo sexo (homens e mulheres).

Na relação entre a aptidão física e a FPM para as mulheres (tabela 2), as aptidões de "força de membros superiores", "agilidade e equilíbrio dinâmico" e "resistência aeróbica" apresentam relação significativa com a FPM direita e esquerda. $\mathrm{O}$ teste de "flexibilidade de membro superior direito" relacionou-se apenas com a FPM direita, enquanto o teste de "agilidade/equilíbrio dinâmico", apenas com a FPM esquerda. 
Tabela 2. Resultados da regressão linear entre a força de preensão manual direita $\left(\mathrm{FPM}_{\mathrm{dir}}\right)$ e esquerda $\left(\mathrm{FPM}_{\text {esq }}\right)$ e os testes de aptidão física em mulheres idosas praticantes de exercícios físicos. FlorianópolisSC, 2011.

\begin{tabular}{lccccc}
\hline \multicolumn{1}{c}{ Variáveis } & $\begin{array}{c}\mathrm{R}^{2} \\
\text { Ajustado }\end{array}$ & $\mathrm{B}$ & $\beta$ & $\mathrm{p}$ & $\begin{array}{c}\text { IC95\% } \\
\text { para B }\end{array}$ \\
\hline Força de membros inferiores (rep) & & & & & \\
$\mathrm{FPM}_{\text {dir }}(\mathrm{kgf})$ & $-0,001$ & 0,053 & 0,092 & 0,347 & $-0,05-0,16$ \\
$\mathrm{FPM}_{\text {esq }}(\mathrm{kgf})$ & 0,034 & 0,137 & 0,207 & $0,033 *$ & $0,01-0,26$
\end{tabular}

Força de membros superiores (rep)

$\begin{array}{llllll}\mathrm{FPM}_{\text {dir }}(\mathrm{kgf}) & 0,057 & 0,213 & 0,258 & 0,008^{*} & 0,05-0,36 \\ \mathrm{FPM}_{\text {esq }}(\mathrm{kgf}) & 0,114 & 0,333 & 0,350 & 0,001^{* *} & 0,16-0,50\end{array}$

lexibilidade de membros inferiores $(\mathrm{cm})$

$\begin{array}{llllll}\mathrm{FPM}_{\text {dir }}(\mathrm{kgf}) & -0,007 & 0,101 & 0,053 & 0,593 & -0,27-0,47 \\ \mathrm{FPM}_{\text {esq }}(\mathrm{kgf}) & -0,003 & 0,176 & 0,079 & 0,418 & -0,25-0,60\end{array}$

Agilidade e equilíbrio dinâmico (seg)

$\begin{array}{llllll}\mathrm{FPM}_{\text {dir }}(\mathrm{kgf}) & 0,067 & -0,056 & -0,276 & 0,004^{*} & -0,09--0,01 \\ \mathrm{FPM}_{\text {esq }}(\mathrm{kgf}) & 0,055 & -0,059 & -0,253 & 0,009 * & -0,10--0,01\end{array}$

Flexibilidade do membro superior direito $(\mathrm{cm})$

$\begin{array}{llllll}\mathrm{FPM}_{\text {dir }}(\mathrm{kgf}) & 0,048 & 0,424 & 0,239 & 0,013^{*} & 0,09-0,75 \\ \mathrm{FPM}_{\text {esq }}(\mathrm{kgf}) & 0,022 & 0,361 & 0,178 & 0,069 & -0,02-0,75\end{array}$

Flexibilidade do membro superior esquerdo $(\mathrm{cm})$

$\begin{array}{llllll}\mathrm{FPM}_{\text {dir }}(\mathrm{kgf}) & 0,015 & 0,276 & 0,155 & 0,112 & -0,06-0,61 \\ \mathrm{FPM}_{\text {esq }}(\mathrm{kgf}) & -0,001 & 0,187 & 0,091 & 0,353 & -0,21-0,58\end{array}$

Resistência aeróbica (m)

\begin{tabular}{llllll}
$\mathrm{FPM}_{\text {dir }}(\mathrm{kgf})$ & 0,141 & 5,530 & 0,386 & $0,001 * *$ & $2,96-8,10$ \\
$\mathrm{FPM}_{\text {esq }}(\mathrm{kgf})$ & 0,145 & 6,447 & 0,391 & $0,001 * *$ & $3,49-9,39$ \\
\hline
\end{tabular}

$\mathrm{FPM}_{\mathrm{dir}}=$ força de preensão manual direita; $\mathrm{FPM}_{\text {ess }}=$ força de preensão manual esquerda; $\mathrm{Kgf}=$ kilograma força; rep= repetição; seg= segundos; $\mathrm{cm}=$ centímetros; $\mathrm{m}=$ metros; $\mathrm{R}^{2}$ Ajustado= variância explicada; $\mathrm{B}=$ inclinação da linha de melhor aderência; coeficiente $\beta=$ inclinação da linha de melhor aderência em desvio-padrão; $\mathrm{p}=$ nível de significância; IC $95 \%$ para B= intervalo de confiança da inclinação da linha de melhor aderência; ${ }^{*} \mathrm{p}<0,05 ;{ }^{* *} \mathrm{p}<0,001$.

Observa-se que, segundo a variância explicada, determinada pelo $\mathrm{R}^{2}$ ajustado, a aptidão "resistência aeróbica" foi mais bem explicada pela variância da FPM, tanto da mão direita, quanto da esquerda (aproximadamente 14\%), seguida do teste de "força de membros superiores", para FPM esquerda (11\%). Porém, ao se observar o valor de B em ambos os testes, verificou-se baixa predição, em que para cada aumento de um desvio-padrão na FPM direita e esquerda, o desempenho nos 
testes aumentou cerca de 0,3 desvio-padrão. Para os demais testes das aptidões "flexibilidade de membro superior direito", "agilidade/equilíbrio dinâmico" e "força de membros inferiores", embora associados significativamente com a FPM, os mesmos apresentaram baixo percentual de explicação e predição (tabela 2).
Entre os homens, observa-se que a FPM relaciona-se com menos testes de aptidão física quando comparados às mulheres. A FPM direita e esquerda associou-se significativamente com a aptidão de "força de membros superiores", "agilidade/equilíbrio dinâmico" e "resistência aeróbica" (tabela 3).

Tabela 3. Resultados da regressão linear entre a força de preensão manual direita $\left(\mathrm{FPM}_{\mathrm{dir}}\right)$ e esquerda $\left(\mathrm{FPM}_{\text {esq }}\right)$ e os testes de aptidão física em homens idosos praticantes de exercícios físicos. FlorianópolisSC, 2011.

\begin{tabular}{|c|c|c|c|c|c|}
\hline Variáveis & $\mathrm{R}^{2}$ Ajustado & $\mathrm{B}$ & $\beta$ & $\mathrm{p}$ & IC95\% para B \\
\hline \multicolumn{6}{|c|}{ Força de membros inferiores (rep) } \\
\hline $\mathrm{FPM}_{\text {dir }}(\mathrm{kgf})$ & $-0,007$ & 0,057 & 0,166 & 0,381 & $-0,07-0,18$ \\
\hline $\mathrm{FPM}_{\mathrm{esq}}(\mathrm{kgf})$ & $-0,031$ & 0,024 & 0,066 & 0,731 & $-0,11-0,16$ \\
\hline \multicolumn{6}{|c|}{ Força de membros superiores (rep) } \\
\hline $\mathrm{FPM}_{\text {dir }}(\mathrm{kgf})$ & 0,221 & 0,216 & 0,497 & $0,005^{*}$ & $0,07-0,36$ \\
\hline $\mathrm{FPM}_{\mathrm{esq}}(\mathrm{kgf})$ & 0,228 & 0,232 & 0,504 & $0,004^{*}$ & $0,07-0,38$ \\
\hline \multicolumn{6}{|c|}{ Flexibilidade de membros inferiores (cm) } \\
\hline $\mathrm{FPM}_{\text {dir }}(\mathrm{kgf})$ & 0,085 & 0,521 & 0,341 & 0,065 & $-0,03-1,07$ \\
\hline $\mathrm{FPM}_{\text {esq }}(\mathrm{kgf})$ & 0,093 & 0,572 & 0,353 & 0,056 & $-0,01-1,15$ \\
\hline \multicolumn{6}{|c|}{ Agilidade e equilíbrio dinâmico (seg) } \\
\hline $\mathrm{FPM}_{\text {dir }}(\mathrm{kgf})$ & 0,210 & $-0,053$ & $-0,487$ & $0,006^{*}$ & $-0,08--0,01$ \\
\hline $\mathrm{FPM}_{\mathrm{esq}}(\mathrm{kgf})$ & 0,199 & $-0,055$ & $-0,476$ & $0,008^{*}$ & $-0,09--0,01$ \\
\hline \multicolumn{6}{|c|}{$\begin{array}{l}\text { Flexibilidade do membro superior direito } \\
(\mathrm{cm})\end{array}$} \\
\hline $\mathrm{FPM}_{\text {dir }}(\mathrm{kgf})$ & $-0,018$ & 0,117 & 0,131 & 0,490 & $-0,22-0,45$ \\
\hline $\mathrm{FPM}_{\mathrm{esq}}(\mathrm{kgf})$ & $-0,016$ & 0,131 & 0,139 & 0,465 & $-0,23-0,49$ \\
\hline \multicolumn{6}{|c|}{$\begin{array}{l}\text { Flexibilidade do membro superior esquerdo } \\
(\mathrm{cm})\end{array}$} \\
\hline $\mathrm{FPM}_{\mathrm{dir}}(\mathrm{kgf})$ & $-0,018$ & 0,128 & 0,129 & 0,496 & $-0,25-0,50$ \\
\hline $\mathrm{FPM}_{\mathrm{esq}}(\mathrm{kgf})$ & $-0,021$ & 0,124 & 0,118 & 0,533 & $-0,27-0,52$ \\
\hline \multicolumn{6}{|l|}{ Resistência aeróbica (m) } \\
\hline $\mathrm{FPM}_{\mathrm{dir}}(\mathrm{kgf})$ & 0,213 & 3,658 & 0,490 & $0,006^{*}$ & $1,13-6,17$ \\
\hline $\mathrm{FPM}_{\mathrm{esg}}(\mathrm{kgf})$ & 0,128 & 3,146 & 0,397 & $0,030^{*}$ & $0,33-5,96$ \\
\hline
\end{tabular}

$\mathrm{FPM}_{\text {dir }}=$ força de preensão manual direita; $\mathrm{FPM}_{\text {esq }}=$ força de preensão manual esquerda; Kgf= kilograma força; rep= repetição; cm= centímetros; $\operatorname{seg}=$ segundos; $\mathrm{m}=$ metros; $\mathrm{R}^{2}$ Ajustado= variância explicada; $\mathrm{B}=$ inclinação da linha de melhor aderência; coeficiente $\beta=$ inclinação da linha de melhor aderência em desvio-padrão; $\mathrm{p}=$ nível de significância; IC95\% para B= intervalo de confiança da inclinação da linha de melhor aderência ${ }^{*} \mathrm{p}<0,05 ;{ }^{*} \mathrm{p}<0,001$. 
Ao analisar a variância explicada, a FPM demonstrou maior explicação (aproximadamente $20 \%$ ) da variância dos testes "agilidade/equilíbrio dinâmico", "resistência aeróbica" e "força de membros superiores", quando comparado às mulheres. Assim, para cada aumento de um desvio-padrão na FPM, essas aptidões aumentam, aproximadamente, meio desvio-padrão.

Observou-se que a FPM é boa preditora da variância da força de membros superiores (aproximadamente 11\%) e da resistência aeróbica nas mulheres (aproximadamente 14\%). Nos homens, a FPM explica a força de membros superiores, resistência aeróbica e agilidade/ equilíbrio dinâmico em aproximadamente $22 \%$, $20 \%$ e $21 \%$, respectivamente.

\section{DISCUSSÃO}

Os resultados do presente estudo apontam que a FPM é preditora da variância da força de membros superiores e da resistência aeróbica nas mulheres e nos homens idosos. Além destas aptidões nos homens, a FPM também explica agilidade/equilíbrio dinâmico. Ao investigar a relação entre a força de preensão manual e as aptidões físicas de idosos ativos, observa-se que a variável "intensidade da modalidade de exercício físico" poderia ter sido considerada. No entanto, essa característica não foi controlada neste estudo, podendo ser considerada um fator limitante, embora se acredite que tal limitação não comprometa os resultados do estudo, uma vez que o foco da pesquisa consiste na predição da FPM sob as aptidões físicas, em idosos praticantes de exercício físico, segundo o sexo.

Estudo realizado sobre a avaliação da força muscular ${ }^{14}$ destaca que esta pode se relacionar à FPM isolada como uma boa preditora, já que as pessoas com pouca força nas mãos também apresentam, geralmente, fraqueza nos demais grupos musculares. A FPM apresentou-se como preditor das causas de mortalidade na população muito idosa e foi considerada uma ferramenta conveniente como prognóstico de risco de morte. ${ }^{15}$
A redução de força muscular no envelhecimento é uma consequência do crescimento de gorduras intramusculares, uma das características da sarcopenia que, em suma, aponta com diminuição da massa muscular, alta taxa de gordura intramuscular e queda de força muscular, que se associam com risco maior de perda de mobilidade. ${ }^{2}$ Portanto, a FPM pode refletir a força muscular global, pois há alta correlação com outras medidas de força, incluindo flexão de braço, extensão do joelho, flexão e extensão do tronco. ${ }^{1}$

Sugeriu-se a utilização da FPM como screening para sarcopenia, podendo indicar prejuízos funcionais futuros, quando se apresenta inferior a 30kgf para os homens e $20 \mathrm{kgf}$ para as mulheres. ${ }^{16}$ Conforme se observou no presente estudo, os valores da FPM apresentaram-se superiores, tanto para os homens como para as mulheres, evidenciando a importância da prática de exercícios físicos na manutenção da funcionalidade do idoso.

A força muscular em idosas saudáveis no período pré-menopausa torna-se importante para a manutenção da saúde óssea. A pouca FPM está relacionada com a baixa densidade mineral óssea da coluna e colo do fêmur, bem como com o aumento do risco de fraturas na pós-menopausa das mulheres coreanas. ${ }^{17}$

Para Barbosa et al., ${ }^{18}$ a FPM é considerada um marcador da força total do indivíduo. Neste estudo, a FPM associou-se com os testes que avaliavam a força, tanto de membros superiores quanto dos inferiores, mas sua predição se mostrou superior no sexo masculino. Pesquisa ${ }^{7}$ apontou que os homens apresentam FPM maior do que as mulheres, em todas as fases do desenvolvimento humano. Alexandre et al. ${ }^{8}$ salientam que tal fato é comum, pois a FPM reflete a massa e a força muscular global, e como os homens possuem maior massa, consequentemente apresentam maior força muscular. Isso ocorre pelo fato de os homens apresentarem maior concentração de hormônios responsáveis pelo turnover proteico 
muscular, como a testosterona, o hormônio do crescimento $(\mathrm{GH})$, entre outros aspectos. ${ }^{8}$

Outro fator relevante é a prática de exercício físico. Belmonte ${ }^{19}$ sugere que sua realização pode influenciar a FPM entre as mulheres, diminuindo as perdas decorrentes da idade. Rebelatto et al. ${ }^{20}$ afirmam que um programa de exercícios físicos regulares pode contribuir para a manutenção da força de preensão bilateral das mãos em mulheres idosas. Quanto aos homens idosos, Belmonte ${ }^{19}$ constatou que a prática de exercício físico parece não influenciar na FPM.

Os estudos de Belmonte ${ }^{19}$ e Rebelatto et al. ${ }^{20}$ levam à hipótese de que as mulheres idosas parecem ter ganho maior na FPM ao praticar determinado exercício físico, quando comparadas aos homens, pois os mesmos já apresentam maior FPM. Desta forma, destaca-se que a intensidade da prática de exercício físico pode ser fator relevante no ganho de FPM em ambos os sexos, necessitando de investigações relacionadas.

Essa predição ainda é pouco estudada, principalmente ao relacionar com cada aptidão física. Wallymahmed et al. ${ }^{21}$ realizaram estudo cujo objetivo foi esclarecer a relação entre a capacidade aeróbica e a FPM com o controle glicêmico em pacientes diabéticos do tipo I, e verificaram que a FPM se correlacionou positivamente com a capacidade aeróbica.

Matsudo $^{22}$ reforça que os valores da FPM têm mostrado associação significativa com a incapacidade funcional, ou seja, indivíduos com menores valores de força apresentaram menor velocidade de andar.

\section{CONCLUSÕES}

Concluiu-se que a força de preensão manual (FPM) é maior nos homens idosos, podendose afirmar que é boa preditora para as aptidões de "força de membros superiores", "agilidade/ equilíbrio dinâmico" e "resistência aeróbica", enquanto que nas mulheres essa predição se mostrou apenas para a "resistência aeróbica". Observou-se que a FPM é boa preditora da força de membros superiores (aproximadamente 11\%) e da resistência aeróbica nas mulheres (aproximadamente 14\%). Nos homens, a FPM explica a força de membros superiores, resistência aeróbica e agilidade/equilíbrio dinâmico em aproximadamente $22 \%, 20 \%$ e $21 \%$, respectivamente.

Ainda que existam algumas limitações, como ausência de um grupo controle, ou padronização de variáveis importantes como o nível de atividade física, o presente estudo apontou relação significativa entre FPM e algumas aptidões físicas. Observou-se também que a FPM prediz apenas a variação de testes físicos que apresentam bom desempenho pelos idosos, o que justifica e aumenta o poder de explicação e predição entre os homens, já que estes obtiveram melhores desempenhos nas avaliações físicas.

Sugere-se que futuros estudos sejam realizados com idosos que praticam exercícios físicos com intensidades diferenciadas, tanto para homens como para mulheres, e que levem em conta as medidas antropométricas no estudo da predição da FPM. Este estudo contribuiu para esclarecer as aptidões físicas (força de membros superiores, agilidade/equilíbrio dinâmico e resistência aeróbia) que são preditas pela força de preensão manual em idosos praticantes de atividade física, já que a maioria das pesquisas envolve apenas idosos frágeis.

Com base nos resultados deste estudo será possível, a partir da mensuração da FPM, identificar alterações nas aptidões, conforme o sexo, promovendo subsídios para que educadores físicos e demais profissionais da saúde possam intervir nos programas de exercícios físicos, de modo que os idosos melhorem essas aptidões físicas. 


\section{REFERÊNCIAS}

1. Sasaki H, Kasagi F, Yamada M, Fujita S. Grip strength predicts cause-specific mortality in middle-aged and elderly persons. Am J Med 2007;120(4):337-42.

2. Arroyo P, Lera L, Sánchez H, Bunout D, Santos JL, Albala C. [Anthropometry, body composition and functional limitations in the elderly]. Rev Méd Chile 2007;135(7):846-54. Espanhol.

3. Kauffman TL. Manual de Reabilitação Geriátrica. Rio de Janeiro: Guanabara Koogan; 2001.

4. Rogatto GP. Força isométrica máxima de indivíduos fisicamente ativos: influência do envelhecimento e do sexo. Lect Educ Fís Deportes 2003;9(67):1-5.

5. Geraldes AAR, De Oliveira ARM, De Albuquerque RB, De Carvalho JM, Farinatti PTV. A força de preensão manual é boa preditora do desempenho funcional de idosos frágeis: um estudo correlacional múltiplo. Rev Bras Med Esporte 2008;14(1):12-6.

6. Benedetti TRB, Meurer ST, Borges LJ, Conceição R, Lopes MA, Morini S. Associação entre os diferentes testes de força em idosos praticantes de exercícios. Fit Perform J 2010;9(1):52-7.

7. Moura PMSL. Estudo da força de preensão palmar em diferentes faixas etárias do desenvolvimento humano [Dissertação]. Brasília: Universidade de Brasília,Faculdade de Ciências da Saúde; 2008.

8. Da Alexandre TS, Duarte YAO, Dos Santos JLF, Lebrão ML. Relação entre força de preensão manual e dificuldade no desempenho de atividades básicas de vida diária em idosos do município de São Paulo. Rev Saúde Coletiva 2008;5(24):178-82.

9. Rikli RE, Jones CJ. Testes de aptidão física para idosos. Barueri: Manole; 2008.

10. Alves RV, Mota J, Costa MC, Alves JGB. Aptidão física relacionada à saúde de idosos: influência da hidroginástica. Rev Bras Med Esporte 2004;10(1):31-7.

11. Rikli RE, Jones CJ. Development and validation of a functional fitness test for community-residing older adults. J Aging Phys Act 1999;7(2):129-81.

12. Bellace JV, Healy D, Besser MP, Byron T, Hohman L. Validity of the dexter evaluation system's Jamar dynamometer attachment for assessment of hand grip strength in a normal population. J Hand Ther 2000;3(1):46-51.
13. Figueiredo IM, Sampaio RF, Mancini MC, Silva FCM, Souza MAP. Teste de força de preensão utilizando o dinamômetro Jamar. Acta Fisiátrica 2007;14(2):104-10.

14. Rebelatto JR, Castro AP, Chan A. Quedas em idosos institucionalizados: características gerais, fatores determinantes e relações com a força de preensão manual. Acta Ortop Bras 2007;15(3):151-4.

15. Ling CHY, Taekema D, De Craen AJM, Gussekloo J, Westendorp RGJ, Maier AB. Handgrip strength and mortality in the oldest old population: the Leiden 85plus study. Can Med Assoc J 2010;182(5):429-35.

16. Lauretani F, Russo CR, Bandinelli S, Bartali B, Cavazzini C, Di Iorio A, et al. Age-associated changes in skeletal muscles and their effect on mobility: an operational diagnosis of sarcopenia. J Appl Physiol 2003;95(5):1851-60.

17. Kim SW, Lee HA, Cho EH. Low handgrip strength is associated with low bone mineral density and fragility fractures in postmenopausal healthy korean Women. J Korean Med Sci 2012;27(7):744-7.

18. Barbosa AR, Souza JMP, Marucci MFN, Lebrão ML. Relação entre estado nutricional e força de preensão manual em idosos do município de São Paulo, Brasil: dados da Pesquisa SABE. Rev Bras Cineantropom Desempenho Hum 2006;8(1):37-44.

19. Belmonte LAO. Análise da força de preensão manual em idosos praticantes e não praticantes de exercícios físicos regulares [dissertação]. Florianópolis: Universidade do Estado de Santa Catarina; 2007.

20. Rebelatto JR, Calvo JI, Orejuela JR, Portillo JC. Influência de um programa de atividade física de longa duração sobre a força muscular manual e a flexibilidade corporal de mulheres idosas. Rev Bras Fisioter 2006;10(1):127-32.

21. Wallymahmed ME, Morgan C, Gill GV, MacFarlane IA. Aerobic fitness and hand grip strength in Type 1 diabetes: relationship to glycaemic control and body composition. Diabet Med 2007;24(11):1296-9.

22. Matsudo SM, Matsudo VKR, Barros Neto TL, De Araújo TL. Evolução do perfil neuromotor e capacidade funcional de mulheres fisicamente ativas de acordo com a idade cronológica. Rev Bras Med Esporte 2003;9(6):365-76. 